\title{
Diacetate Cellulose-Silicon Bionanocomposite Adsorbent for Recovery of Heavy Metal Ions and Benzene Vapours: An Experimental and Theoretical Investigation
}

\author{
Akhror Yarkulov 1(D), Bakhrom Umarov ${ }^{2(D)}$, Feruza Rakhmatkarieva ${ }^{3(D)}$, Nuritdin Kattaev 4 (D), \\ Khamdam Akbarov 5 (D), Elyor Berdimurodov ${ }^{3, *}$ \\ 1 Faculty of Chemistry, National University of Uzbekistan, Tashkent, 100034, Uzbekistan; yaaxror@ rambler.ru (A.Y.); \\ 2 Faculty of Chemistry, National University of Uzbekistan, Tashkent, 100034, Uzbekistan; umarovbahrom57@mail.ru \\ (B.U.); \\ 3 Institute of General and Nonorganic Chemistry, Academy of the Science of the Republic of Uzbekistan, Tashkent city, \\ 100180, Uzbekistan; rakhfi@yandex.ru (F.R.); elyor170690@gmail.com (E.B.); \\ 4 Faculty of Chemistry, National University of Uzbekistan, Tashkent, 100034, Uzbekistan; ntkattaev@ gmail.com (N.K.); \\ 5 Faculty of Chemistry, National University of Uzbekistan, Tashkent, 100034, Uzbekistan; Kh_akbarov@mail.ru (K.A.); \\ * Correspondence: elyor170690@gmail.com (E.B.);
}

Received: 10.05.2021; Revised: 15.06.2021; Accepted: 20.06.2021; Published: 8.08.2021

\begin{abstract}
The diacetate cellulose-silicon bionanocomposite adsorbent (DACSBNC) was first introduced to recover heavy metal ions and benzene vapors. The adsorption thermodynamics of heavy metal ions and benzene vapors on the DACSBNC was first investigated by the adsorption-calorimetric, $\mathrm{X}$-ray, and theoretical methods. The observed results confirmed that (i) the adsorption capacitance of DACSBNC for cadmium (II), mercury (II), and lead (II) ions were accounted for 12.23, 13.87, and $31.40 \mathrm{mg} / \mathrm{g}$, respectively; (ii) the sorption capacitance of DACSBNC for benzene vapors was 0.5618 $\mathrm{mmol} / \mathrm{g}$. The quantum chemical calculation was also carried out on the density functional theory (DFT) using the 6-31G $(\mathrm{d}, \mathrm{p}) / \mathrm{B} 3 \mathrm{LYP}$ basis sets in Gaussian 09. The results of the quantum chemical analysis support the experimental results.
\end{abstract}

Keywords: cellulose diacetate; silica; bionanocomposite; sorption isotherm; DFT.

(C) 2021 by the authors. This article is an open-access article distributed under the terms and conditions of the Creative Commons Attribution (CC BY) license (https://creativecommons.org/licenses/by/4.0/).

\section{Introduction}

At present times, the research on natural polysaccharides is an interesting topic; because they are natural compounds, non-toxicity, biodegradability, and biocompatibility [1-3] The polysaccharides are using to syntheses the nanostructured hybrid materials (bionanocomposites) [4]. The bionanocomposites are bio-based polymer compounds widely applied in the pharmaceutical, biomedical, and chemical industries [5].

Currently, cellulose and its derivatives are combined with inorganic substances in mineralization, resulting in a new type of material, which is named the bionanocomposites [67]. It is noted that the natural minerals are mainly bionanocomposites. The biopolymers combined with inorganic compounds to form the bionanocomposites in the biomimizing processes [8]. Cellulose can be modified with inorganic compounds by the vapor or aqueous phase [9]. The sol-gel methods are performed to modify the cellulose with the inorganic compounds. The conductive, antimicrobial, hydrophilicity, and hydrophobicity properties of natural cellulose polymer were improved by modifying inorganic substances [10]. 
Additionally, the production of bionanocomposites with new functionality and characteristics was also increased with the modification of the inorganic compounds [11]. In addition to this, the cellulose and its derivatives also have unique properties such as good permeability, selectivity, hydrophilicity, thermal and chemical stability, mechanical strength, low cost, which makes it a good raw material for the in situ synthesis of nanomaterials by sol-gel technology [12-13]. The sol-gel method is a more effective way to obtain the organic-inorganic bionanocomposites based on the natural polysaccharides [14]. In this method, the organicinorganic bionanocomposites were synthesized by the hydrolysis and polycondensation of various alkoxysilanes in a polysaccharide matrix, forming an inorganic phase of silicon oxide [15]. The sizes of silica particles depend on the following parameters: the synthesis temperature, $\mathrm{pH}$ values of the medium, nature of organic solvents, reaction ratio between the alcohol-silane and water, the type and amount of the catalyst [16].

Sol-gel synthesis products are used in the biology, environment, energy, mechanics, ionics, electronics [17], and optics as the biosensors, catalysts, functional smart coatings, fuel and solar cells, membranes, and separation devices, sorbents, bionanocomposites, sensors for the separation of aqueous-organic mixtures, wastewater treatment from heavy metal ions and anionic dyes, as well as for biomedical purposes [18].

Using the bionanocomposites adsorbents for heavy metal and organic compounds is the most interesting investigation [19]. The adsorption properties of bionanocomposites are more favourable than the traditional nanocompounds [20]. The adsorbent performance and selectivity properties depend on the pore size, the volume of the sorption space, specific surface area, and degree of dispersion of bionanocomposites [21].

\section{Materials and Methods}

\subsection{Adsorption analysis.}

Adsorption-calorimetric investigations were performed on a universal high-vacuum volumetric installation. The volumetric-liquid method and an isothermal differential automatic microcalorimeter of the Tiana-Calvet were used to measure the values of adsorbed compounds on the bionanocomposite [22].

\subsection{Thermodynamic calculations based on the theory of volumetric filling of micropores} (TVFM) equation.

The adsorption processes occurred on the microporous zeolites related to the bulk filling mechanism. The TVFM method was performed to calculate the adsorption degree $(\theta)$ according to Equation 1 [23].

$$
\theta=\exp \left[-(A / E)^{n}\right]
$$

where $n$ is the independent parameter and $E$ is adsorption energy. The $n$ exponent depends on the structure of the adsorbent [24]. $A$ is the work of adsorption at $\mathrm{kJ} / \mathrm{mol}$, which transfers 1 mole of gas from the surface of a liquid adsorbate (pressure $P^{\circ}$ ) into an equilibrium gas phase (pressure $P$ ) related to Equation 2.

$$
A=R \operatorname{Tln}\left(P^{\circ} / P\right)
$$

The adsorption value $(a)$ can be represented as the following Equation 3:

$$
a=a_{o} \exp \left[-(A / E)^{n}\right]
$$


Equation 3 is the general equation of the theory of volumetric filling of micropores (TVFM). In logarithmic form, it has a linear form (Equation 4):

$$
\ln a=\ln a_{0}-(A / E)^{n}
$$

where $a$ is adsorption value in micropores at $\mathrm{mmol} / \mathrm{g} ; a_{0}$ is the adsorption limit at $\mathrm{mmol} / \mathrm{g} ; A$ is differential molar work of adsorption at $\mathrm{kJ} / \mathrm{mol}$ [25].

The wide distribution of micropores was measured from Equation 5:

$$
a=\left(W_{01} / V^{*}\right) \exp \left[-\left(A / E_{1}\right)^{2}\right]+\left(W_{02} / V^{*}\right) \exp \left[-\left(A / E_{2}\right)\right]
$$

where $E_{1}$ and $E_{2}$ are adsorption energies at the first and second adsorption layer; $W_{01}$ and $W_{02}$ expressing adsorption in micropores at the first and second adsorption layer. The values of $E_{1}$ was over $E_{2}$ for supermicropores [26]. In this equation, $\mathrm{V}^{*}$ is the molar volume of the adsorbed substance. Parameters of $W_{01}, E_{1}, W_{02}$ and $E_{2}$ are easily determined from one experimental adsorption isotherm for a wide range of equilibrium relative pressures; for example, $1 \cdot 10^{-6}$ $\div 5 \cdot 10^{-1}$ p. Parameters with index 1 are characteristics of the microporous structure, and those with index 2 are characteristics of the supermicroporous structure of the adsorbent [27].

\subsection{Thermogravimetric analysis.}

A derivate-graph system I. Paulik, F. Paulik, and L. Erdey brand Q-1500 (Hungary) were performed to analyze the thermal properties of the studied samples. The studied samples were dried to achieve the absolute mass before the experiment. Then, the dried form was made small to achieved the dispersed state [28]. Next, it was dried again in a drying oven at $70-105^{\circ} \mathrm{C}$ to remove residual moisture and solvents to obtain the constant weight. A prepared sample with a weighed portion of $10 \mathrm{mg}$ was placed in a ceramic crucible and heated in a derivate-graph system at an air atmosphere pressure. The temperature ranged from room temperature to $800^{\circ} \mathrm{C}$ with a heating rate of $50 \mathrm{C} / \mathrm{min}$ [29]. Alumina was used as a comparatively inert substance (carrier). The analysis was carried out according to the TG (thermogravimetry) and DTA (differential thermogravimetry) curves, which were obtained by recording on photographic paper. The obtained thermograms were used to determine the thermal-oxidative stability [1].

\subsection{Investigation of samples by $X$-ray phase analysis.}

X-ray studies of the samples were carried out on a Panalytical Empyrean X-ray diffractometer equipped with a $\mathrm{Cu}$ tube $(\mathrm{K} \alpha 1=1.5406 \AA)$. The measurements were carried out at room temperature in the $2 \theta$ range of angles, in the range from $5^{\circ}$ to $80^{\circ}$, and in the step-bystep scanning mode with a step of 0.007 degrees.

\subsection{Spectrophotometric method.}

The spectrophotometric method (spectrophotometer EMC-30PC-UV, EMC Labs Germany) was employed to measure the concentration of various heavy metals $\left(\mathrm{Pb}^{2+}, \mathrm{Cd}^{2+}\right.$, and $\mathrm{Hg}^{2+}$ ). The quantitative determination of the components of a mixture is based on the fact that the optical density of any mixture is equal to the sum of the optical densities of the individual components. Wavelength for the determination of $\mathrm{Pb}^{2+}, \mathrm{Cd}^{2+}$ and $\mathrm{Hg}^{2+}$ were 510, 495 and $500 \mathrm{~nm}$, respectively [28]. 


\section{Results and Discussion}

\subsection{Adsorption and thermodynamics of DACSBNC with benzene.}

A universal high-vacuum volumetric setup was used to investigate the adsorption characteristics, making it possible to research with high accuracy. Before starting the experiment, the benzene vapors were pumped out at $10^{-5}-10^{-6} \mathrm{~Pa}$ for 10 hours.

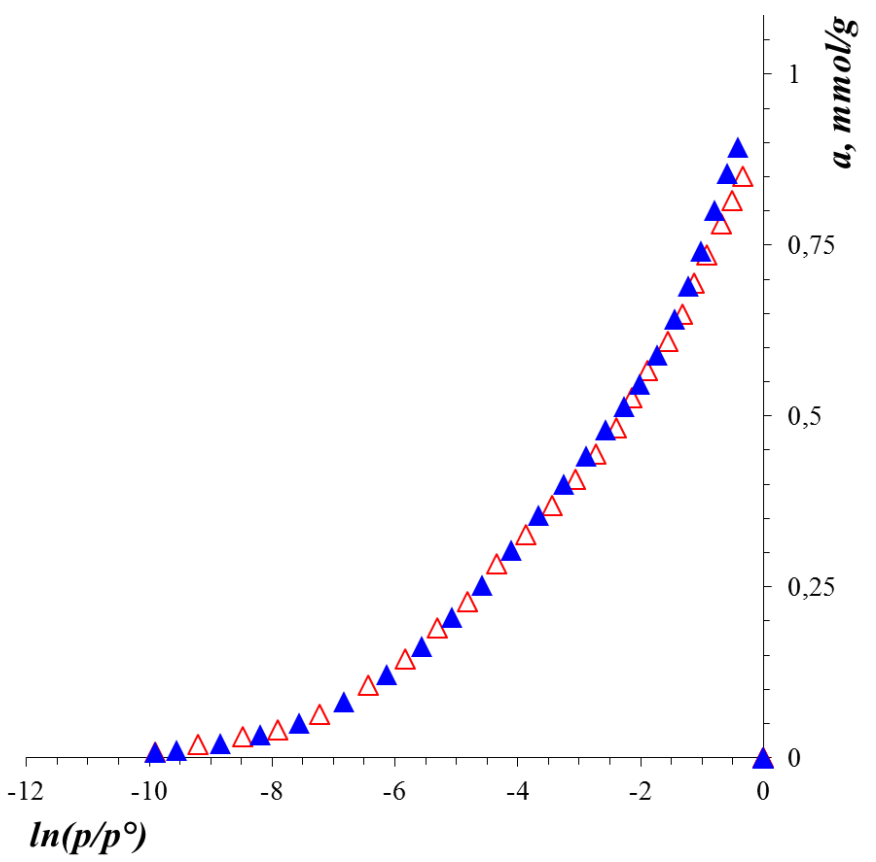

Figure 1. Isotherm of benzene vapors on DACSBNC at $303 \mathrm{~K}$. Blue dots - calculated using TVFM.

The adsorption isotherm of benzene in semilogarithmic coordinates on DACSBNC at a component ratio of 50:50 in the presence of a citric acid (LA) is shown in Figure 1. It is depicted from Figure 1 that the $\mathrm{P} / \mathrm{P}^{\mathrm{o}}$ was $0.00073\left(\ln \mathrm{P} / \mathrm{P}^{\mathrm{o}}=-7.22\right)$, demonstrating that the adsorption centers are more active and strongly interacted with benzene vapors. The reason for this is that the benzene vapors are effectively adsorbed on DACSBNC by forming hydrophobic bonds between the adsorbent and benzene vapors. In addition to this, the sorption capacitance of DACSBNC for benzene vapors was rose to $0.923 \mathrm{mmol} / \mathrm{g}$ at $\mathrm{P} / \mathrm{P}^{\circ}=0.98$.

Figure 1 also shows benzene's theoretically calculated adsorption isotherm on DACSBNC, which are indicated by blue triangles. The two-term TVFM Equation 6 indicated the adsorption isotherm of benzene vapors on the DACSBNC:

$$
\mathrm{a}=0,639 \exp [-(\mathrm{A} / 11,99)]^{2}+0,299 \exp [-(\mathrm{A} / 2,84)]^{2}
$$

The data calculated by Equation 6 are in good agreement with the experimental data. Equation parameters: the proportion of the first term a $01=0.639 \mathrm{mmol} / \mathrm{g}, \mathrm{E}_{01}=11.99 \mathrm{~kJ} / \mathrm{mol}$, $\mathrm{n}_{1}=2$; the fraction of the second term $\mathrm{a}_{02}=0.299 \mathrm{mmol} / \mathrm{g}, \mathrm{E}_{02}=2.84 \mathrm{~kJ} / \mathrm{mol}, \mathrm{n}_{2}=2$. The two-term TVFM equation describes the main adsorption region with high exponent values under the exponential, demonstrating a high homogeneity of the sorption system. TVFM assumes two mechanisms of adsorption: firstly, around $68 \%$ of the sorption space in the DACSBNC has filled benzene vapors at normal pressure; secondly, another part of sorption space was filled with benzene vapor when the pressure increase. 


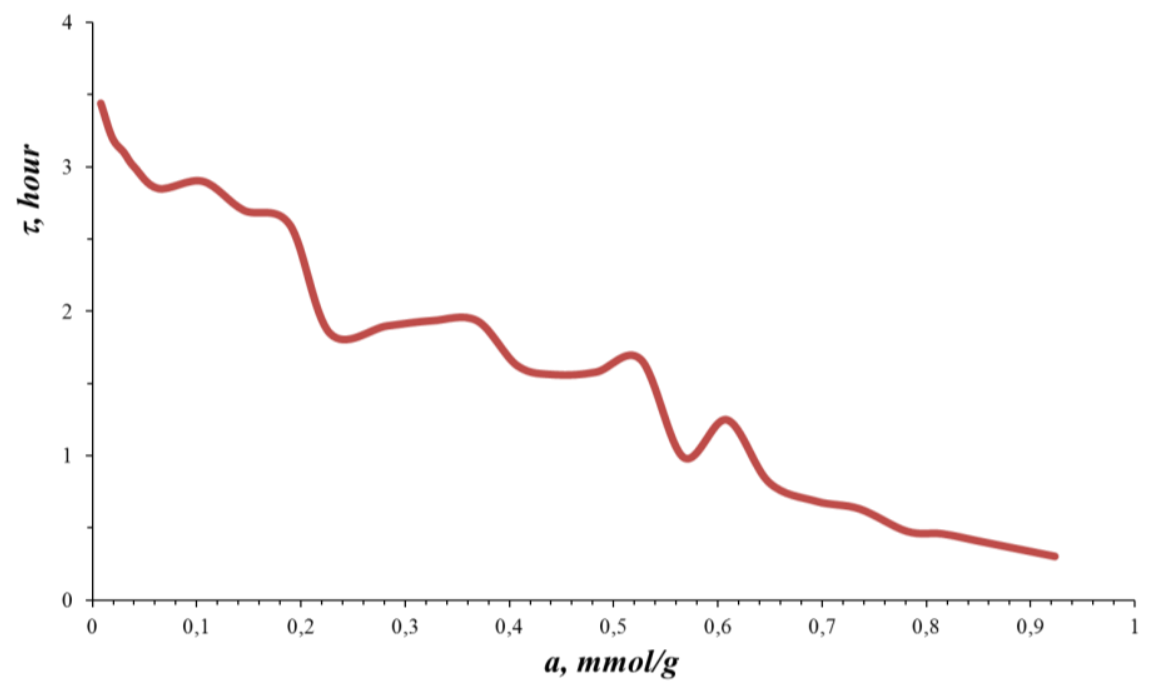

Figure 2. The correlation between time and adsorption of benzene vapors on DACSBNC.

The correlation between time and adsorption of benzene vapors on DACSBNC was indicated in Figure 2. It is demonstrated that the adsorption equilibrium was established at 3.44 hours. On an average of 1.85 hours, the adsorption reached equilibrium in the biggest hydrophobic centers ( 2 units). At the first stage (long adsorption equilibrium time), the strong hydrophobic bonds between the benzene vapors and DACSBNC in the adsorption active sites were formed. At the next stage (around 1 hour), the rigid adsorbate - adsorbent bonds were formed.

Figure 3 indicated that the differential heats of adsorption of benzene vapors on DACSBNC at $303 \mathrm{~K}$ : horizontal dashed line is the heat of condensation of benzene vapors. The values of adsorption heats were measured with high accuracy using the method of compensating heat fluxes by the Peltier. The found adsorption isotherm (Figure 3) confirmed that the differential heats of adsorption curves were 5 sections, which are differed from the $91.30 \mathrm{~kJ} / \mathrm{mol}$.

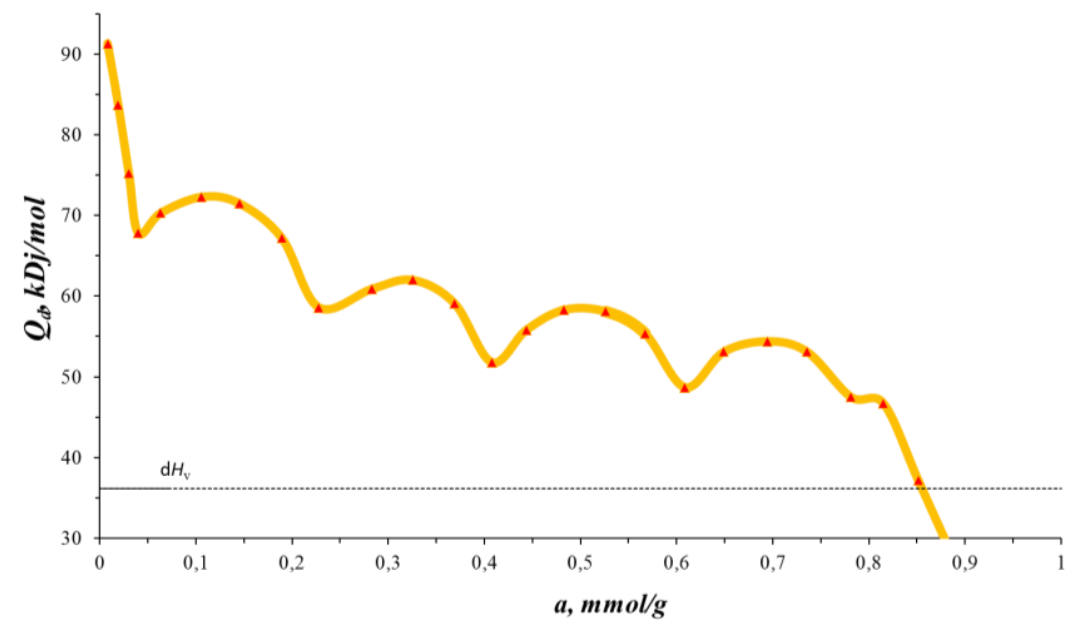

Figure 3. Differential heats of adsorption of benzene vapors on DACSBNC at $303 \mathrm{~K}$ : horizontal dashed line is the heat of condensation of benzene vapors.

It is clearly described in Figure 3 that the differential heats of adsorption curves were 5 sections; the difference in the 5 sections was around $0.2 \mathrm{mmol} / \mathrm{g}$. These 5 stages also show the number of hydrophobic centers in DACSBNC, which are responsible for the adsorption of benzene vapors. Figure 3 also revealed the excellent interaction between the benzene vapors 
and DACSBNC. This interaction depends on the molecular structure of the studied DACSBNC. The benzene vapors are linked to the DACSBNC by the hydrophobic centers.

The values of benzene adsorption on the DACSBNC in the first hydrophobic region varies within the range of $0-0.04 \mathrm{mmol} / \mathrm{g}$ and are accompanied by a sharp drop in heat 91.30 $67.80 \mathrm{~kJ} / \mathrm{mol}$. The next region has low hydrophobic energy; the values of benzene adsorption on the DACSBNC were accounted for between 0.04 and $0.228 \mathrm{mmol} / \mathrm{g}$. In this stage, the adsorption heat of benzene vapors on the DACSBNC was reduced from 67.80 to $58.60 \mathrm{~kJ} / \mathrm{mol}$. In the third region, the adsorption heat of benzene vapors on the DACSBNC decreased from 58.60 to $51.80 \mathrm{~kJ} / \mathrm{mol}$ while the values of benzene adsorption on the DACSBNC rose from 0.228 to $0.408 \mathrm{mmol} / \mathrm{g}$. At the following region, the adsorption heat of benzene vapors on the DACSBNC decreased from 51,80 to $48.70 \mathrm{~kJ} / \mathrm{mol}$ while the values of benzene adsorption on the DACSBNC increased from 0.408 to $0.609 \mathrm{mmol} / \mathrm{g}$. In the last region, the adsorption heat of benzene vapors on the DACSBNC decreased from 48.70 to $47.50 \mathrm{~kJ} / \mathrm{mol}$ while the values of benzene adsorption on the DACSBNC increased from 0.609 to $0.780 \mathrm{mmol} / \mathrm{g}$. It is also found that the condensation heat of benzene was $36.13 \mathrm{~kJ} / \mathrm{mol}$ on the surface of the DACSBNC.

The values in the differential molar entropy of adsorption $\left(\Delta \mathrm{S}_{\mathrm{d}}\right)$ of benzene vapors on the DACSBNC were calculated using the Gibbs - Helmholtz equation (Figure 4). It is underlined that the entire entropy plot is cited below the entropy level of liquid benzene and grows in a wave-like manner with an increase in the filling of the bionanocomposition. The entropy of benzene adsorption on the DACSBNC in the first region rose from -114.70 to -7.02 $\mathrm{J} / \mathrm{mol} \cdot \mathrm{K}$ while the adsorption values achieved the minimum amount $(0.019-0.106 \mathrm{mmol} / \mathrm{g}) . \mathrm{It}$ is noted that the average values of integral entropy in the adsorption of benzene vapors on the DACSBNC accounted for $-33.94 \mathrm{~J} / \mathrm{mol} \cdot \mathrm{K}$; this value is importantly lower than the entropy of solid benzene, showing the blocked state of benzene vapors in the pores of the DACSBNC.

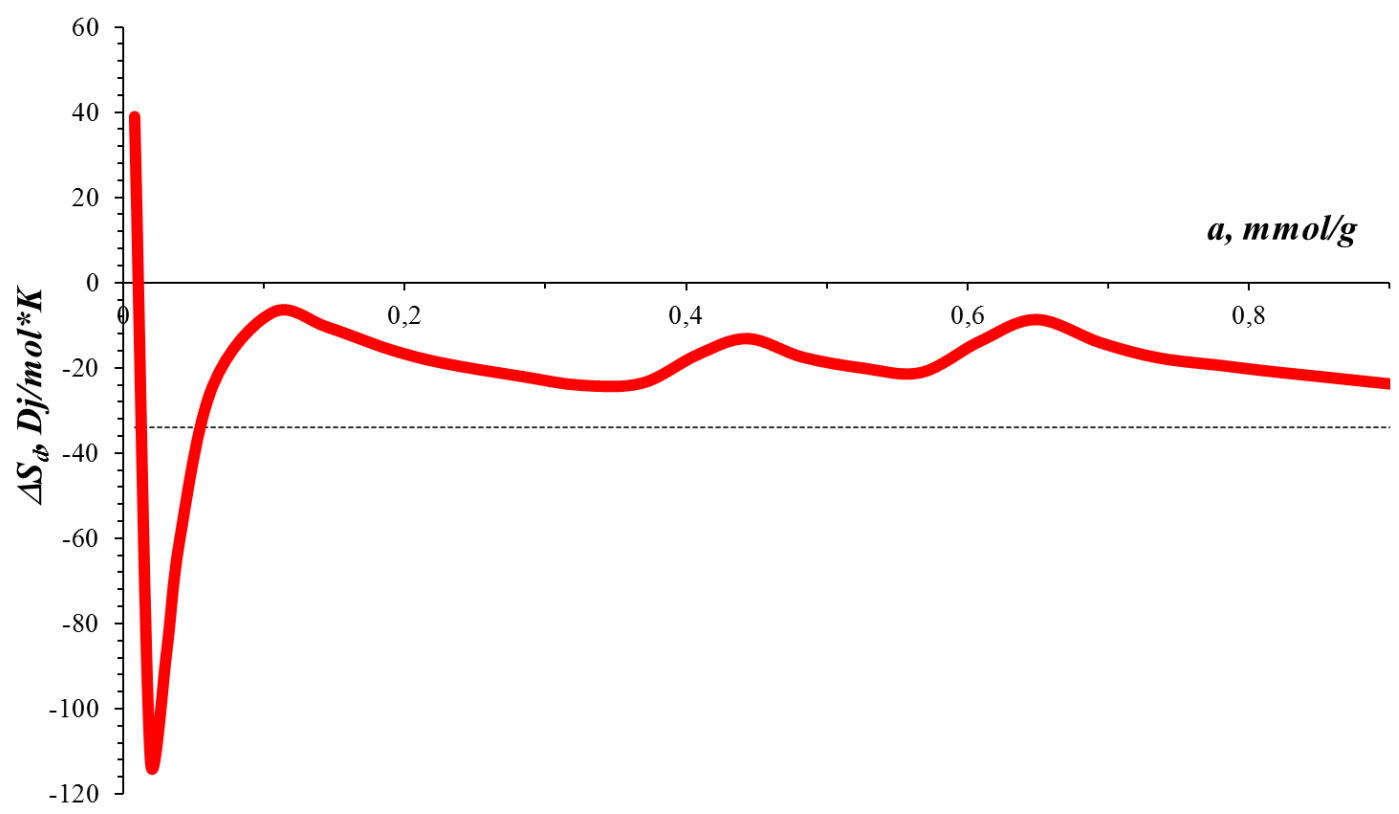

Figure 4. The entropy of benzene vapors adsorption in benzene vapors on DACSBNC at $303 \mathrm{~K}$ : horizontal dashed line - mean integral entropy; entropy of liquid benzene is taken to be zero.

\subsection{Thermal analysis of DACSBNC.}

The stability of the adsorbent is an important factor. The stability of benzene vapors adsorption in benzene vapors on DACSBNC was examined with the thermal analysis. Therefore, the study of their thermal properties is relevant. To study thermooxidative 
degradation, thermogravimetric curves of DAC samples and DACSBNC were researched. For each thermogravimetric curve, the kinetics of thermal-oxidative destruction was calculated. Information on the composition and results of thermogravimetric analysis of DACSBNC samples is given in Table 1.

Table 1. Data on the composition and results of thermogravimetric analysis of samples.

\begin{tabular}{|c|c|c|c|c|c|}
\hline \multirow{2}{*}{ Sample } & \multicolumn{3}{|c|}{ The molar ratio of the starting components } & \multirow[t]{2}{*}{$\begin{array}{c}\text { Loss of } \\
\text { masses. \% }\end{array}$} & \multirow{2}{*}{$\begin{array}{c}\text { The remainder, } \\
\text { masses. } \%\end{array}$} \\
\hline & TEOC & DAC & LK & & \\
\hline $10 / 90$ & 1 & 1,00 & - & 74 & 26 \\
\hline $20 / 80$ & 1 & 0,60 & - & 68 & 32 \\
\hline $30 / 70$ & 1 & 0,03 & - & 15 & 85 \\
\hline $40 / 60$ & 1 & 0,03 & 0,10 & 18 & 82 \\
\hline $50 / 50$ & 1 & 0,10 & - & 34 & 66 \\
\hline $60 / 40$ & 1 & 0,10 & 0,10 & 38 & 62 \\
\hline $70 / 30$ & 1 & 0,30 & - & 52 & 48 \\
\hline $80 / 20$ & 1 & 0,30 & 0,10 & 56 & 44 \\
\hline $90 / 10$ & 1 & 0,30 & - & 48 & 34 \\
\hline
\end{tabular}

The temperature of the onset of the destruction of samples of DAC-silica bionanocomposites obtained in acetic acid, as shown in Figures 5-8, is also near the onset of DAC destruction.

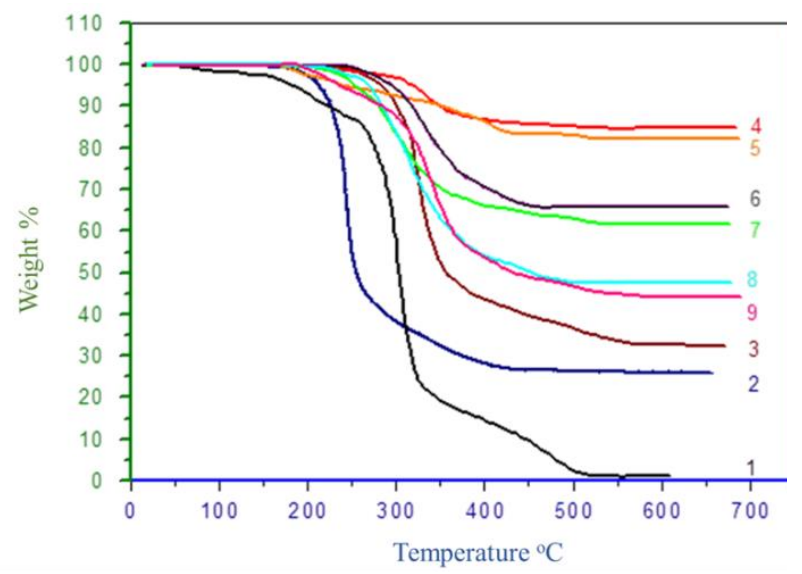

Figure 5. Thermograms: DAC (1) and hybrids $10 / 90$ (2); 20/80 (3), 30/70 (4), 40/60 (5); 50/50 (6), 60/40 (7), 70/30 (8) and 80/20 (9)

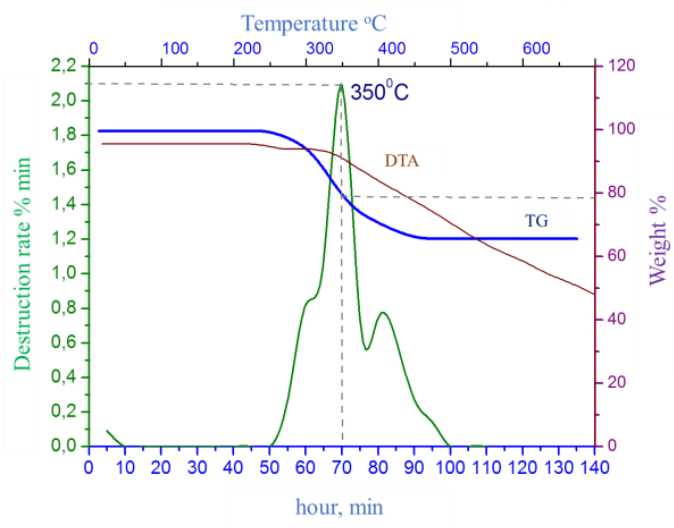

Figure 7. Derivatogram of the hybrid bionanocomposite material DAC-silica 50/50

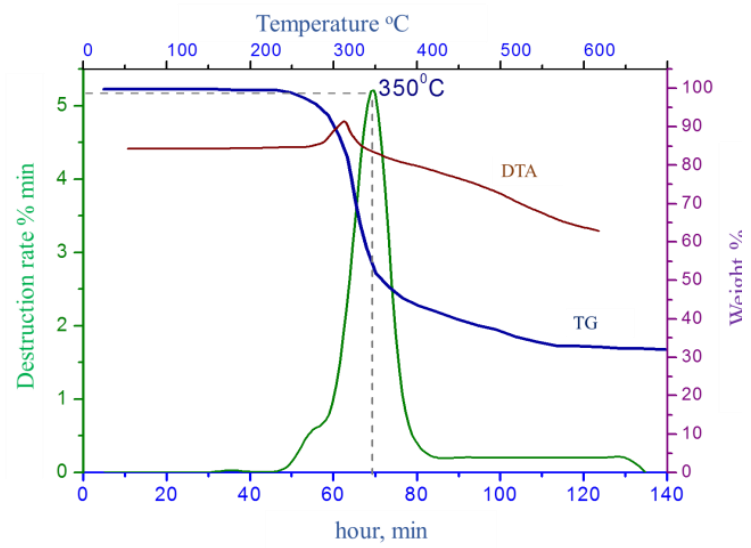

Figure 6. Derivatogram of a hybrid bionanocomposite material DAC-silica 20/80

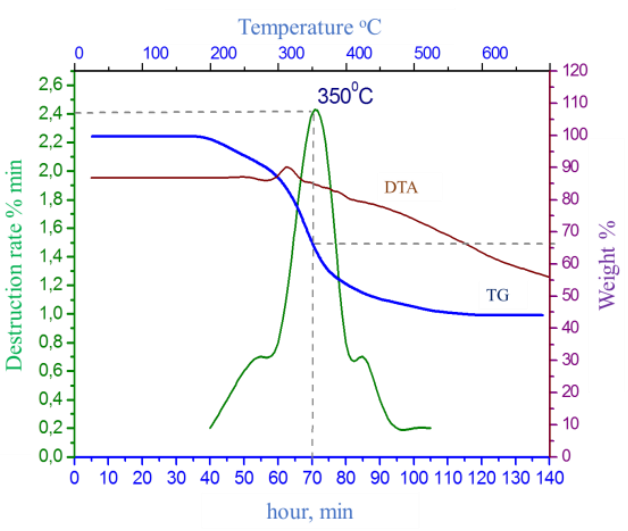

Figure 8. Derivatogram of the hybrid bionanocomposite material DAC-silica 80/20

These data show that the highest rates of destruction at temperature maxima are exhibited by hybrids 1 and 2 synthesized from the TEOS / DAC / $\mathrm{HCl}$ reaction mixtures without using "reaction" water, i.e., no added ethanol. They approach the maximum rate of DAC 
destruction. However, the activation energies for hybrids 10/90 and 20/80 exceed those for DAC by $\sim 2.3$ and 1.9 times, respectively.

The hybrid DAC-silica bionanocomposite was synthesized in the presence of citric acid. This is due to the citric acid can influence to reach maximum reaction yield in the hybrid DAC-silica bionanocomposite syntheses.

As can be seen from these graphs, the rate of thermal-oxidative destruction of samples of DAC-silica bionanocomposites depends on the content of DAC, with an increase in which the rate of destruction increases. The same applies to the activation energy. But, in the presence of citric acid, there is a tendency to decrease the activation energy in each specific case.

Thus, the results of studying the thermooxidative destruction of samples synthesized by the sol-gel method at various molar ratios of the initial TEOS, DAC, and various additives showed that the kinetic characteristics: the maximum rate and activation energy of thermooxidative destruction are influenced by the content of DAC in the initial reaction mixtures and the presence of added substances.

\subsection{X-ray phase analysis of DACSBNC.}

The important parameters of processes in the substances on the surface and interface of phases were investigated with the $\mathrm{X}$-ray phase analysis. The sorption-structural characteristics (strength, particle size, pore diameter, size surface area) of DACSBNC was found.

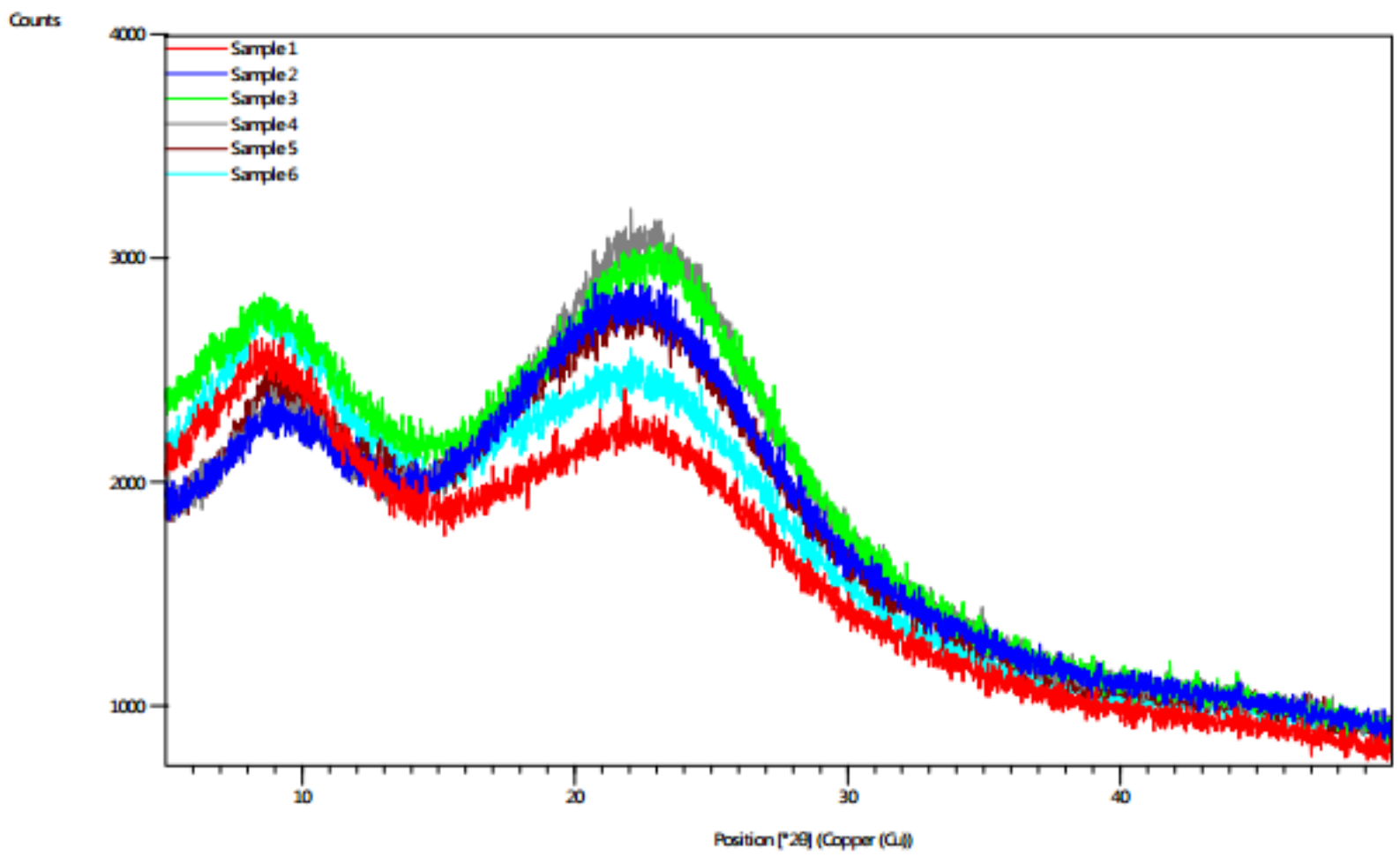

Figure 9. X-ray diffraction patterns of DACSBNC of various compositions: 1. (60:40) + LC; 2. (50:50) + LC; 3 . $(40: 60)+$ LC; 4.40:60; 5.50:50; 6. 60:40.

X-ray phase analysis of DACSBNC showed two extrema (Figure 9); the first at 4-130 and the second at 15-350, typical for amorphous materials. X-ray diffraction in the samples shows that the amorphous material contains silicon dioxide and carbon, with possible bonds between silicon and polysaccharide. When DACSBNC interacts with silica, the silica network expands, while the addition of LA changes the DACSBNC confirmation and, accordingly, the interplanar distances $\mathrm{d}$ also differ. 
This indicates a partial suppression of the crystallinity of DACSBNC in the structure of the hybrid material, i.e., on the relationship of DACSBNC macromolecules with nanoparticles formed as a result of hydrolytic polycondensation of silica. A sharp peak $2 \theta$ is observed at 230, which corresponds to amorphous $\mathrm{SiO}_{2}$.

\subsection{Quantum-chemical calculations of hybrid bionanocomposite materials cellulose} diacetate-silica.

Computer modeling methods provide valuable information not only about the structure and physicochemical properties of compounds but also useful for predicting their chemical properties [30-33].

In this regard, a quantum chemical study of intermolecular interactions in cellulose diacetate-silica was carried out within the density potential theory (DFT) framework. The initial cellulose diacetate-silica model was built and pre-optimized using the Avogadro and GaussView programs. Quantum-chemical calculations were performed by using the Gaussian 09 program package within the framework of density functional theory (DFT) employing 6$31 \mathrm{G}(\mathrm{d}, \mathrm{p})$ basis set and Beckes three-parameter (local, nonlocal, Hartee-Fock) hybrid exchange functionals with Lee-Yang-Parr correlation functionals (B3LYP) [34-35].

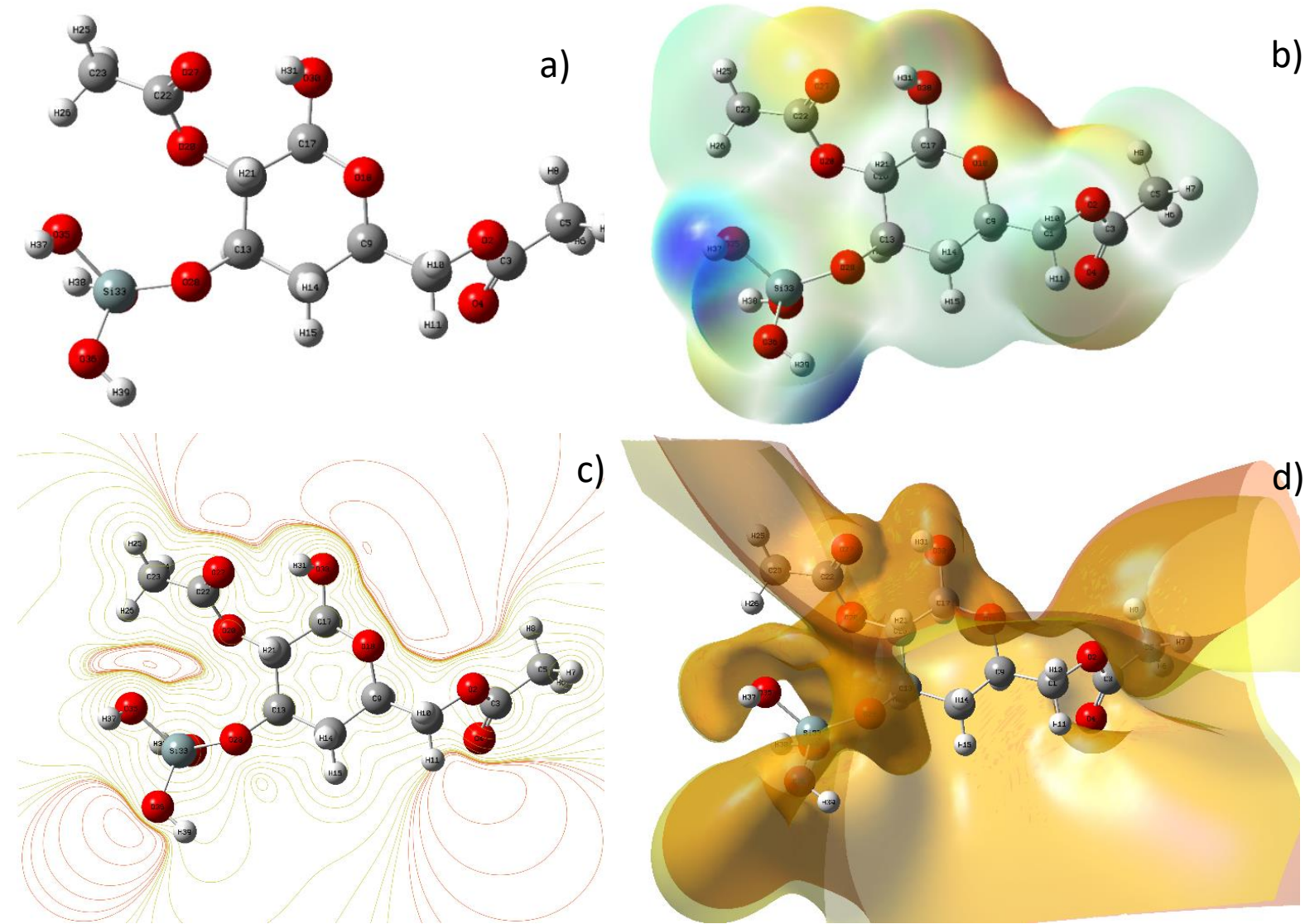

Figure 10. The molecular geometry of cellulose diacetate-silica composite optimized at B3LYP/6-31G(d,p) level (a) ${ }^{1}$, the molecular electrostatic potential (MEP) surface (b), and the contour map of MEP surface (c) and the free surface upon excitation state (d) of the elementary unit of a cellulose diacetate-silica composite.

${ }^{1}$ Optimized structural parameters, such as bond lengths $(\AA)$ and bond angles $\left(^{\circ}\right)$ calculated by DFT method using 6-31G $(\mathrm{d}, \mathrm{p})$ basis set, is given in Tables $\mathrm{S} 1$ (supporting information)

Figure 10 shows the molecular geometry of cellulose diacetate-silica composite optimized at B3LYP/6-31G(d,p) level (Figure 10a), the molecular electrostatic potential (MEP) surface (Figure 10b), and the contour map of MEP surface (Figure 10c) and the free surface 
upon excitation state (Figure 10d) of the elementary unit of a cellulose diacetate-silica composite [36-38].

These maps allow us to visualize the variably charged regions of the molecule. The molecular electrostatic potential (MEP) surface shows a molecule's positively and negatively charged electrostatic potential. A red color with a negative value indicates a minimum electrostatic potential (meaning it is weakly bound or has excess electrons) and acts as an electrophilic agent. The blue color indicates the maximum electrostatic potential, which acts the other way around (Figure 10b).

To see all the surfaces of the MEP, you can simply draw each surface as an outline around the molecule. As can be seen from Figure 10c, each contour around the molecule is an MEP surface; the outer contour has a lower isosurface value, and the inner contour has a higher isosurface value (Figure 10c); Figure 10d demonstrate the free surface available upon excitation of the elementary unit of cellulose diacetate-silica.

The frontier molecular orbitals theory is the simplest version of the perturbation theory in the molecular orbital method (MOM), which is designed to predict the reactivity of compounds. For this purpose, the so-called reaction molecules are considered. Frontier molecular orbitals (FMOs) - the highest occupied molecular orbital (HOMO/HOMO), which acts as an electron donor, and the lowest unoccupied/molecular orbital (LUMO/LUMO), which acts as an electron acceptor, are very important parameters for quantum chemistry.

In this case, the region with the highest boundary density of the lowest free orbital, as a rule, is most favorable for a nucleophilic attack; the electrophilic attack proceeds mainly at the region where the boundary density of the highest occupied orbital is greatest. Interaction with charge transfer between the highest occupied orbital of one reactant and the lowest free orbital of the other leads to an increase in the frontiers density in the overlap region.

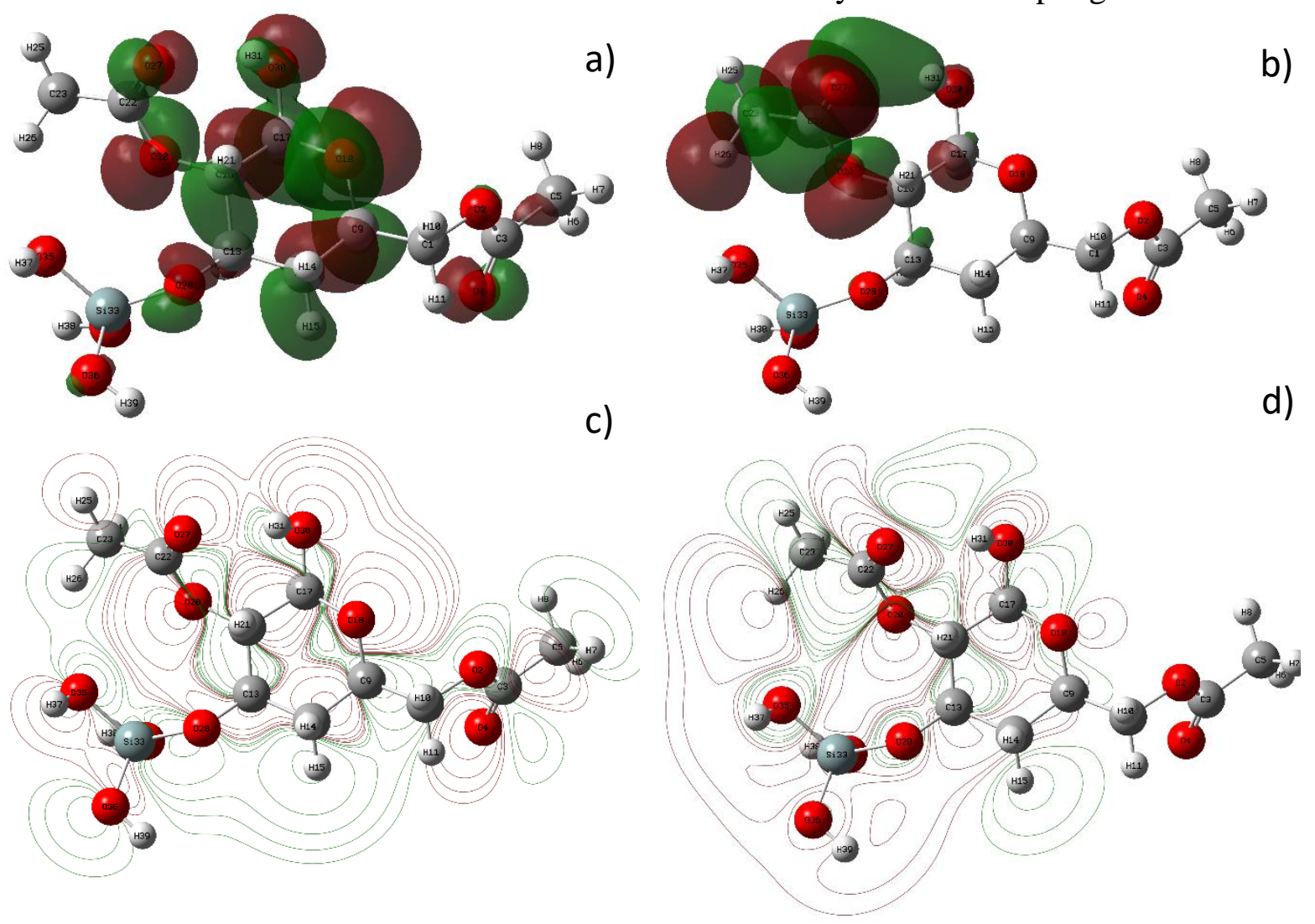

Figure 11. FMOs (HOMO-LUMO) $(a, b)$ and their contours $(c, d)$ for the elementary unit of the DACSBNC. 
To describe the reactivity, it is only necessary to know the form of the boundary MO of the reacting molecules, which, as a rule, is determined using quantum chemical calculations.

When the bandgap is small, the molecule is highly polarizable and highly reactive. DFT calculations provide important information about the selectivity region of the molecular framework. Еномо and Elumo, which clarify the nature of the interaction of charge exchange within the macromolecule.

Figure 11 shows the FMOs and their contours of the elementary unit of the cellulose diacetate-silica macromolecule. As mentioned above, HOMO acts as an electron donor, and LUMO acts as an acceptor. In radicals or excited molecules, the role of one of these orbitals or both of them can be played by singly occupied MOs. The most important characteristic of boundary MOs is their partial electron density, i.e., the density of individual atoms called the boundary electron density or reactivity index.

The main postulate of the theory: reactions proceed most easily in the case of maximum overlap of FMOs, which, as a rule, make the greatest contribution to the interaction energy of the reactants. Overlapping leads to charge transfer from the highest occupied donor orbital to the lowest free acceptor.

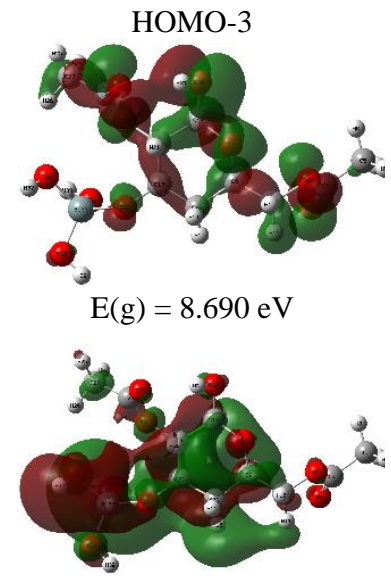

$\mathrm{LUMO}+3$
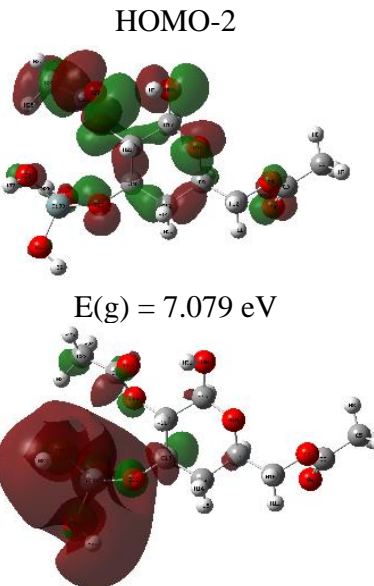

$\mathrm{LUMO}+2$

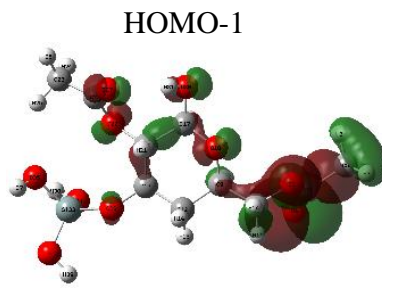

$\mathrm{E}(\mathrm{g})=6.318 \mathrm{eV}$

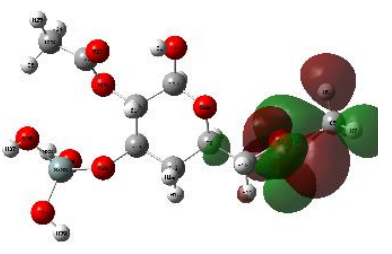

LUMO+1

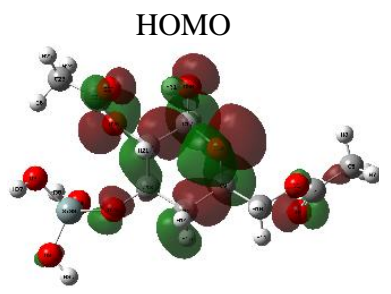

$\mathrm{E}(\mathrm{g})=3.678 \mathrm{eV}$

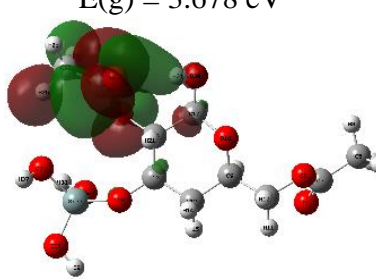

LUMO

Figure 12. FMOs (HOMO-LUMO) and bandgap energies for an elementary unit of a DACSBNC.

The bandgap E ( $\mathrm{g}$ ) of the molecule is $3.678 \mathrm{eV}$, the frontier energies of the molecular orbitals Еномо and Elumo are -4.479 and $-0.801 \mathrm{eV}$, respectively (Figures 11 and 12). The low energy of the forbidden zone makes it possible to assert that the system is polar, kinetically unstable, and chemically active.

Table 2. Some parameters for DACSBNC calculated by DFT method using 6-31G (d, p) basis set.

\begin{tabular}{l|c} 
Parameters $(\mathrm{eV})$ & Value \\
\hline Eномо & -4.7170 \\
\hline ELUмо & -2.4960 \\
\hline Energy gap $\left[\mathrm{E}(\mathrm{g})=\mathrm{E}_{\mathrm{LUMO}}-\mathrm{E}_{\text {HOмо }}\right]$ & 2.2210 \\
\hline Dipole moment $(\mu)$ & 2.7444 Debye \\
\hline Ionization energy $(\mathrm{I})$ & 4,7170 \\
\hline Electron affinity $(\mathrm{A})$ & 2,4960 \\
\hline Electronegativity $(\chi)$ & 3,6065 \\
\hline Chemical potential $\left(\mu_{\mathrm{o}}\right)$ & $-3,6065$ \\
\hline Hardness $(\eta)$ & 1,1105 \\
\hline Global softness $(\varsigma)$ & 0,4502 \\
\hline Global electrophilicity $(\omega)$ & 5,8563
\end{tabular}


Some parameters such as the global softness $(\varsigma)$, global electrophilicity $(\omega)$, hardness $(\eta)$, chemical potential $(\mu \mathrm{o})$, ionization potential $(\mathrm{I})$, electronegativity $(\chi)$, electron affinity $(\mathrm{A})$, energy gap (E (g)), HOMO and LUMO energies and dipole moment $(\mu)$ for the cellulose diacetate-silica have been calculated at B3LYP / 6-31G (d, p) basis set. The results are given in Table 2.

The electrophilicity, global hardness, and chemical potential will help in many ways to understand the structure of the molecules and their reactivity with the aid of DFT-based descriptors. As shown in Table 2, the chemical potential is negative, and it means that cellulose diacetate-silica is stable.

3.5. The use of a sorbent with hybrid cellulose diacetate-silica bionanocompositions for purification of source waters from heavy metal ions.

Recently, attention has been drawn to functionalized nanoporous hybrid nanocomposites, for the synthesis of which one-stage sol-gel or template methods are used, based on the reaction of hydrolytic polycondensation. Using these methods, it is possible to obtain a broad palette of sorbents that differ in their morphology, textural and mechanical characteristics.

In this work, for the purification of wastewater from heavy metal ions using a DACSBNC, we propose a sorption-spectrophotometric method for the determination of lead (II), mercury (II), and cadmium (II). The optimal conditions for the sorption extraction of heavy metal ions (cadmium (II), mercury (II), and lead (II)) by a hybrid bionanocompositional cellulose diacetate-silica sorbent have been determined.

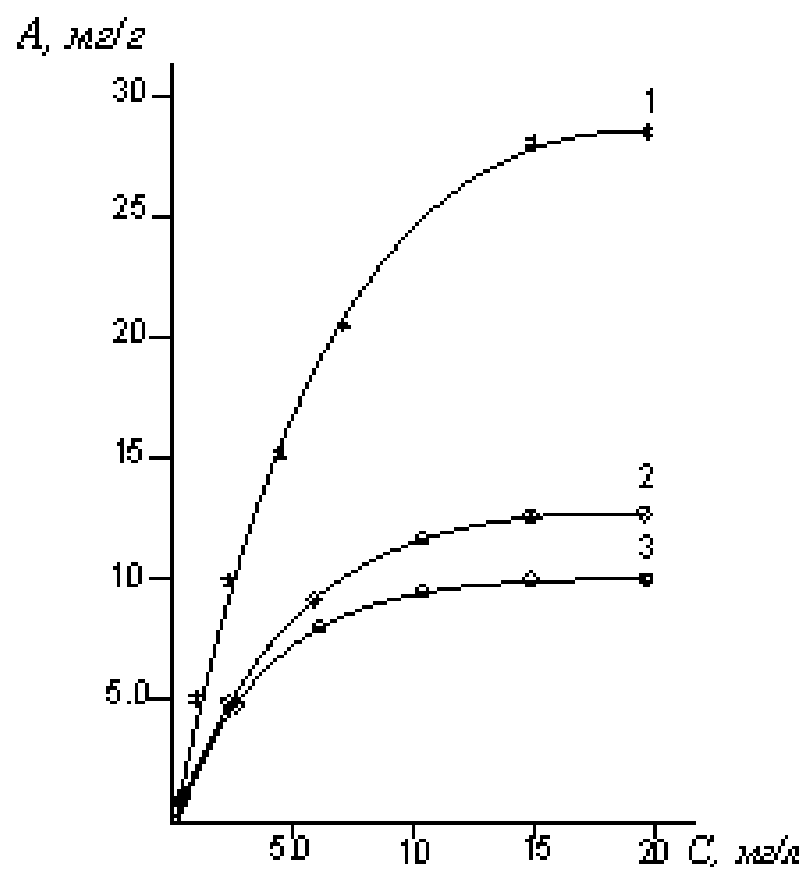

Figure 13. Sorption isotherms of (1) $\mathrm{Pb}$ (II); (2) $\mathrm{Hg}$ (II); (3) Cd (II) on the DACSBNC.

It is clear from Figure 13 that the sorption isotherms of (1) $\mathrm{Pb}$ (II); (2) $\mathrm{Hg}$ (II); (3) $\mathrm{Cd}$ (II) on the diacetate cellulose-silicon bionanocomposite adsorbent. To describe sorption isotherms, the Langmuir Equation 7was used in the following form: 


$$
\mathrm{a}=\mathrm{am} \mathrm{m}_{\mathrm{d}}\left(\mathrm{C} / \mathrm{C}_{0}\right) / 1+\mathrm{K}_{\mathrm{d}}\left(\mathrm{C} / \mathrm{C}_{0}\right)
$$

where $a$ is the adsorption value ( $\mathrm{mol} / \mathrm{g}$ ), $a_{m}$ is the capacity of the adsorption monolayer or the maximum adsorption value $(\mathrm{mol} / \mathrm{g}), C$ is the equilibrium concentration $(\mathrm{mol} / \mathrm{l}), C_{0}$ is the standard concentration (mol/l), $K_{d}$ is the equilibrium constant the process of interaction of the adsorbate with the adsorbent (Langmuir's constant). Usually, for the values of $K_{d}$ and am, the Langmuir Equation 8 leads to the following form:

$$
1 / \mathrm{a}=1 / \mathrm{am}+1 \cdot \mathrm{C} / \mathrm{K}_{\mathrm{d}} \cdot \mathrm{a}_{\mathrm{m}} \cdot \mathrm{C}
$$

A graph of $1 /$ a versus $1 / \mathrm{C}$ is plotted, the tangent of the slope of this straight line gives the value $1 / \mathrm{K}_{\mathrm{d}} \mathrm{am}$, and the segment cut off from the ordinate axis is $1 / \mathrm{am}$.

The straight-line nature of this dependence indicates that this process is subordinate to the laws of the monomolecular theory of adsorption.

The standard Gibbs energy of adsorption $\Delta \mathrm{G}^{0}$ ads was determined from the dependence of the equilibrium constants on $298 \mathrm{~K}$, which are given in Table 3.

Sorption studies were carried out under static conditions. The values of the maximum capacity of the sorbents have been calculated. Calculations have shown that sorption is high at initial concentrations, and equilibrium is established with increased concentration.

The constants of sorption equilibrium $\left(\mathrm{K}_{\mathrm{d}}\right)$, the limiting capacities of the monolayer $(\mathrm{am})$, and the standard Gibbs energy of adsorption $\left(\Delta \mathrm{G}^{0}\right.$ ads $)$ of the DACSBNC sorbent for heavy metal ions were calculated (Table 3).

Table 3. Adsorption values are presented in coordinates of the linear form of the Langmuir equation.

\begin{tabular}{l|l|l|l} 
Metall & $\mathrm{Cd}^{2+}$ & $\mathrm{Hg}^{2+}$ & $\mathrm{Pb}^{2+}$ \\
\hline$K_{d}$ & 21,10 & 21,46 & 47,17 \\
\hline$a_{m}$ & 16,14 & 17,15 & 45,02 \\
\hline$-\Delta G^{0}$ ads. $k D j / m o l$ & 7,555 & 7,597 & 9,548
\end{tabular}

The value of the constant adsorption equilibrium $K_{d}$ characterizes the distribution of metals between the adsorbent and water. The higher the value of the constant, the better it extracts metals from the solution. Experimental calculations using the Langmuir equation showed that the values of the equilibrium constants and the limiting adsorption decrease in the following series: $\mathrm{Pb}^{2+}>\mathrm{Hg}^{2+}>\mathrm{Cd}^{2+}$. The location of such a sequence can depend on the ionic radius of the metals.

Table 3 shows that the value of the standard Gibbs energy takes negative values for the adsorption of all metals. If the standard Gibbs energy gets negative values, the process can proceed spontaneously. Consequently, the adsorption of metals on the DACSBNC is a spontaneous process.

The results of calculating the effect of temperature on the capacity of DACSBNC sorbents for heavy metal ions of DACSBNC sorbents are presented in Table 4.

Table 4. Influence of temperature on the sorption capacity of the DACSBNC sorbent

\begin{tabular}{l|c|c|c|c} 
Sorbent & $\mathrm{Me}$ & $\mathrm{Pb}^{2+}$ & $\mathrm{Hg}^{2+}$ & $\mathrm{Cd}^{2+}$ \\
\hline \multirow{4}{*}{ HBNMDAC } & $\mathrm{t},{ }^{\circ} \mathrm{C}$ & \multicolumn{3}{|c}{$\mathrm{A}, \mathrm{mg} / \mathrm{g}$} \\
\cline { 2 - 5 } & 20 & 31,40 & 13,87 & 12,23 \\
\cline { 2 - 5 } & 40 & 20,25 & 11,14 & 11,14 \\
\cline { 2 - 5 } & 60 & 17,47 & 8,15 & 9,08
\end{tabular}

From Table 4, it can be seen that with increasing temperature in the equilibrium state, the capacity of the sorbent decreases, which indicates that the process obeys the laws of physical adsorption. 
It is possible that at the beginning of the sorption process, heavy metal ions are adsorbed on the surface until it is completely filled, forming a monomolecular layer, and then, with an increase in concentration, begin to penetrate inside, filling the amorphous zones of structures obtained from DACSBNC.

Table 5. Sorption capacity of sorbents based on cellulose and its hybrid bionanocompositions.

\begin{tabular}{c|c|c} 
№ & Samples & Sorption capacity \\
\hline 1 & Cellulose hybrid - zirconium dioxide & Ni (II) $4.95, \mathrm{Fe}$ (III) $0.89, \mathrm{Zr}$ (IV) $0.70, \mathrm{Cu}$ (II) $0.58, \mathrm{Cd}$ (II) $0.57, \mathrm{Cr}$ \\
& $\left(\mathrm{ZrO}_{2}\right)$ & $\mathrm{Co}$ (III) $0.36 \mathrm{mg} / \mathrm{g}$ \\
\hline 2 & Cellulose acetate hybrid- silica & $\mathrm{Cr}$ (VI) $19.46 \mathrm{mg} / \mathrm{g}$ \\
\hline 3 & Cellulose-COOH & $\mathrm{Cu}$ (II) $2.24, \mathrm{Zn}$ (II) $0.13 \mathrm{mg} / \mathrm{g}$ \\
\hline 4 & Cottoncellulose & $\mathrm{Cd}$ (II) $57.12, \mathrm{Cu}$ (II) $14.72, \mathrm{Fe}$ (II) $11.2, \mathrm{Ni}$ (II) 9.28 and $\mathrm{Zn}$ (II) 7.8 \\
& silica & $\mathrm{Cr}$ (VI) $1.08 \mathrm{mg} / \mathrm{g}$
\end{tabular}

Table 5 for comparison shows the values of cellulose's sorption capacities and its hybrid bionanocompositions. It can be seen that for cellulose, the sorption capacity takes small values, and for nanohybrids, the sorption capacity increases sharply.

The desorption of metal ions from saturated sorbents of DACSBNC with solutions of mineral acids with increasing temperature has been investigated. The optimal conditions for the process have been determined. It was found that the best eluent for heavy metal ions is diluted $0.5 \mathrm{M}$ nitric acid, while the degree of extraction is $90-98 \%$.

Sorption of heavy metal ions $\mathrm{Pb}^{2+}, \mathrm{Hg}^{2+}$, and $\mathrm{Cd}^{2+}$ from wastewater is a monomolecular process and obeys the Langmuir equation. The constants of sorption equilibrium, the limiting capacities of the monolayer, and the values of the standard Gibbs energy of the sorbent based on hybrid bionanocompositions for heavy metal ions are calculated.

The obtained research results showed that the sorbent based on hybrid bionanocomposite materials cellulose diacetate-silica is the most selective for $\mathrm{Pb}$ (II) ions and the least selective for Cd (II) ions.

\section{Conclusion}

For adsorption isotherms of DACSBNC obtained by precision adsorption-calorimetry, two-term equations of the theory of volumetric filling of micropores are recommended, which describe well the processes taking place.

Differential heats of adsorption of benzene in DACSBNC have a stepwise form. Benzene is adsorbed with high heat, large negative values of the mean integral entropy are observed, which is explained by strong retardation of the mobility of adsorbate molecules, which slows down the establishment of adsorption equilibrium.

The physicochemical properties of DACSBNC have been studied by various methods depending on the conditions of the sol-gel process, the results of which are recommended to be used for the controlled synthesis of nanocomposite materials with the required nanoporous structure and functionality.

Quantum-chemical calculations within the density functional theory (DFT) framework showed low bandgap energy, which suggests that the system is polar kinetically unstable and chemically active.

The investigated DACSBNC materials have been proposed as promising sorbents for the extraction of heavy toxic metal ions such as lead (II), mercury (II), and cadmium (II) from galvanic aqueous solutions. 


\section{Funding}

This research received no external funding.

\section{Acknowledgments}

This work was supported by a project of the Ministry of Innovative Development of the Republic of Uzbekistan (project No. F7-54).

\section{Conflicts of Interest}

The authors declare no conflict of interest.

\section{References}

1. Joshi, N.C.; Malik, S.; Gururani, P. Utilization of Polypyrrole/ZnO Nanocomposite in the Adsorptive Removal of $\mathrm{Cu} 2+, \mathrm{Pb} 2+$ and $\mathrm{Cd} 2+$ Ions from Wastewater. Letters in Applied NanoBioScience 2021, 10, 23392351, https://doi.org/10.33263/LIANBS103.23392351.

2. Sharma, A.; Singh, M.; Arora, K.; Singh, P.P.; Badru, R.; Singh Kang, T.; Kaushal, S. Preparation of cellulose acetate-Sn(IV) iodophosphate nanocomposite for efficient and selective removal of $\mathrm{Hg} 2+$ and $\mathrm{Mn} 2+$ ions from aqueous solution. Environmental Nanotechnology, Monitoring \& Management 2021, 16, 100478, https://doi.org/10.1016/j.enmm.2021.100478.

3. Naushad, M.; Ahamad, T.; Al-Sheetan, K.M. Development of a polymeric nanocomposite as a high performance adsorbent for $\mathrm{Pb}(\mathrm{II})$ removal from water medium: Equilibrium, kinetic and antimicrobial activity. J. Hazard. Mater. 2021, 407, 124816, https://doi.org/10.1016/j.jhazmat.2020.124816.

4. Mahato, B.N.; Krithiga, T.; Mary Thangam, M.A. Rapid adsorption of As(V) from aqueous solution by $\mathrm{ZnO}$ embedded in mesoporous aluminosilicate nanocomposite adsorbent: Parameter optimization, kinetic, and isotherms studies. Surfaces and Interfaces 2021, 23, 100636, https://doi.org/10.1016/j.surfin.2020.100636.

5. Zhang, H.; Xu, F.; Xue, J.; Chen, S.; Wang, J.; Yang, Y. Enhanced removal of heavy metal ions from aqueous solution using manganese dioxide-loaded biochar: Behavior and mechanism. Sci. Rep. 2020, 10, 6067, https://doi.org/10.1038/s41598-020-63000-z.

6. Mishra, S.; Singh, A.K.; Singh, J.K. Ferrous sulfide and carboxyl-functionalized ferroferric oxide incorporated PVDF-based nanocomposite membranes for simultaneous removal of highly toxic heavy-metal ions from industrial ground water. J. Membr. Sci. 2020, 593, 117422 , https://doi.org/10.1016/j.memsci.2019.117422.

7. Deng, J.; Li, X.; Wei, X.; Liu, Y.; Liang, J.; Song, B.; Shao, Y.; Huang, W. Hybrid silicate-hydrochar composite for highly efficient removal of heavy metal and antibiotics: Coadsorption and mechanism. Chem. Eng. J. 2020, 387, 124097, https://doi.org/10.1016/j.cej.2020.124097.

8. Nematidil, N.; Nezami, S.; Mirzaie, F.; Ebrahimi, E.; Sadeghi, M.; Farmani, N.; Sadeghi, H. Fabrication and characterization of a novel nanoporous nanoaerogel based on gelatin as a biosorbent for removing heavy metal ions. J. Sol-Gel Sci. Technol. 2021, 97, 721-733, https://doi.org/10.1007/s10971-020-05439-0.

9. Javadian, H.; Ruiz, M.; Taghvai, M.; Sastre, A.M. Novel magnetic nanocomposite of calcium alginate carrying poly(pyrimidine-thiophene-amide) as a novel green synthesized polyamide for adsorption study of neodymium, terbium, and dysprosium rare-earth ions. Colloids Surf. Physicochem. Eng. Aspects 2020, 603, 125252, https://doi.org/10.1016/j.colsurfa.2020.125252.

10. Liu, J.; Chen, T.-W.; Yang, Y.-L.; Bai, Z.-C.; Xia, L.-R.; Wang, M.; Lv, X.-L.; Li, L. Removal of heavy metal ions and anionic dyes from aqueous solutions using amide-functionalized cellulose-based adsorbents. Carbohydr. Polym. 2020, 230, 115619, https://doi.org/10.1016/j.carbpol.2019.115619.

11. Yu, P.; Wang, X.; Zhang, K.; Wu, M.; Wu, Q.; Liu, J.; Yang, J.; Zhang, J. Continuous purification of simulated wastewater based on rice straw composites for oil/water separation and removal of heavy metal ions. Cellulose 2020, 27, 5223-5239, https://doi.org/10.1007/s10570-020-03135-4.

12. Putri, K.N.A.; Keereerak, A.; Chinpa, W. Novel cellulose-based biosorbent from lemongrass leaf combined with cellulose acetate for adsorption of crystal violet. Int. J. Biol. Macromol. 2020, 156, 762-772, https://doi.org/10.1016/j.ijbiomac.2020.04.100.

13. Zhan, C.; Sharma, P.R.; He, H.; Sharma, S.K.; McCauley-Pearl, A.; Wang, R.; Hsiao, B.S. Rice husk based nanocellulose scaffolds for highly efficient removal of heavy metal ions from contaminated water. Environmental Science: Water Research \& Technology 2020, 6, 3080-3090, https://doi.org/10.1039/D0EW00545B.

14. Na, Y.; Lee, J.; Lee, S.H.; Kumar, P.; Kim, J.H.; Patel, R. Removal of heavy metals by polysaccharide: a review. Polymer-Plastics Technology and Materials 2020, 59, 1770-1790, https://doi.org/10.1080/25740881.2020.1768545. 
15. Sharma, G.; Naushad, M. Adsorptive removal of noxious cadmium ions from aqueous medium using activated carbon/zirconium oxide composite: Isotherm and kinetic modelling. J. Mol. Liq. 2020, 310, 113025, https://doi.org/10.1016/j.molliq.2020.113025.

16. Iqhrammullah, M.; Marlina, M.; Khalil, H.P.S.A.; Kurniawan, K.H.; Suyanto, H.; Hedwig, R.; Karnadi, I.; Olaiya, N.G.; Abdullah, C.K.; Abdulmadjid, S.N. Characterization and Performance Evaluation of Cellulose Acetate-Polyurethane Film for Lead II Ion Removal. Polymers 2020, 12, https://doi.org/10.3390/polym12061317.

17. Isawi, H. Using Zeolite/Polyvinyl alcohol/sodium alginate nanocomposite beads for removal of some heavy metals from wastewater. Arabian Journal of Chemistry 2020, 13, 5691-5716, https://doi.org/10.1016/j.arabjc.2020.04.009.

18. Ramalingam, B.; Parandhaman, T.; Choudhary, P.; Das, S.K. Biomaterial Functionalized GrapheneMagnetite Nanocomposite: A Novel Approach for Simultaneous Removal of Anionic Dyes and Heavy-Metal Ions. ACS Sustainable Chemistry \& Engineering 2018, 6, 6328-6341, https://doi.org/10.1021/acssuschemeng.8b00139.

19. Choi, K.; Lee, S.; Park, J.O.; Park, J.-A.; Cho, S.-H.; Lee, S.Y.; Lee, J.H.; Choi, J.-W. Chromium removal from aqueous solution by a PEI-silica nanocomposite. Sci. Rep. 2018, 8, 1438, https://doi.org/10.1038/s41598-018-20017-9.

20. Abdellatif, F.H.H.; Abdellatif, M.M. Bio-based i-carrageenan aerogels as efficient adsorbents for heavy metal ions and acid dye from aqueous solution. Cellulose 2020, 27, 441-453, https://doi.org/10.1007/s10570-01902818-X.

21. Karami, S.; Zeynizadeh, B. Reduction of 4-nitrophenol by a disused adsorbent: EDA-functionalized magnetic cellulose nanocomposite after the removal of Cu2+. Carbohydr. Polym. 2019, 211, 298-307, https://doi.org/10.1016/j.carbpol.2019.01.113.

22. Hassan, H.; Salama, A.; El-Ziaty, A.K.; El-Sakhawy, M. New chitosan/silica/zinc oxide nanocomposite as adsorbent for dye removal. Int. J. Biol. Macromol. 2019, 131, 520-526, https://doi.org/10.1016/j.ijbiomac.2019.03.087.

23. Tomonaga, H.; Tanigaki, Y.; Hayashi, K.; Matsuyama, T.; Ida, J. Adsorption properties of poly(NIPAM-coAA) immobilized on silica-coated magnetite nanoparticles prepared with different acrylic acid content for various heavy metal ions. Chem. Eng. Res. Des. 2021, 171, 213-224, https://doi.org/10.1016/j.cherd.2021.05.005.

24. Taddesse, A.M.; Ketema, T.T.; Teju, E. Cellulose acetate-Sn (IV) molybdophosphate: A biopolymer supported composite Exchanger for the removal of selected heavy metal ions. Bull. Chem. Soc. Ethiop. 2020, 34, 259-276, https://dx.doi.org/10.4314/bcse.v34i2.5.

25. Liu, S.; Cui, S.; Guo, H.; Wang, Y.; Zheng, Y. Adsorption of Lead Ion from Wastewater Using Non-Crystal Hydrated Calcium Silicate Gel. Materials 2021, 14, https://doi.org/10.3390/ma14040842.

26. Almasian, A.; Giahi, M.; Chizari Fard, G.; Dehdast, S.A.; Maleknia, L. Removal of heavy metal ions by modified PAN/PANI-nylon core-shell nanofibers membrane: Filtration performance, antifouling and regeneration behavior. Chem. Eng. J. 2018, 351, 1166-1178, https://doi.org/10.1016/j.cej.2018.06.127.

27. Meera, K.S.; Arunbabu, D. Magnetic Cellulose Green Nanocomposite Adsorbents for the Removal of Heavy Metal Ions in Water/Wastewater. In Green Biopolymers and their Nanocomposites, Springer: 2019; 423-437, https://doi.org/10.1007/978-981-13-8063-1_18.

28. Zare, E.N.; Motahari, A.; Sillanpää, M. Nanoadsorbents based on conducting polymer nanocomposites with main focus on polyaniline and its derivatives for removal of heavy metal ions/dyes: A review. Environ. Res. 2018, 162, 173-195, https://doi.org/10.1016/j.envres.2017.12.025.

29. Elmorsy, E.E.; Abdelghany, A.M.; Ayad, D.M.; El Gammal, O.A. Synthesis and Physicochemical Studies of Polyvinyl Alcohol Polymer Modified with Copper Thiosemicarbazide Complex. Lett. Appl. NanoBioScience 2021, 10, 2624-2636, https://doi.org/10.33263/lianbs104.26242636.

30. Elyor Berdimurodov, Abduvali Kholikov, Khamdam Akbarov, Guobao Xu, Aboubakr M. Abdullah, Morteza Hosseini, New anti-corrosion inhibitor (3ar,6ar)-3a,6a-di-ptolyltetrahydroimidazo[4,5-d]imidazole-2,5(1 h,3h)-dithione for carbon steel in $1 \mathrm{M} \mathrm{HCl}$ medium: gravimetric, electrochemical, surface and quantum chemical analyse. Arab. J. Chem. 2020, 13, 7504-7523. https://doi.org/10.1016/j.arabjc.2020.08.025

31. A.R. Shahmoradi, M. Ranjbarghanei, A.A. Javidparvar, L. Guo, E. Berdimurodov, Bahram Ramezanzadeh, Theoretical (atomic-DFT\&molecular-MD), surface/electrochemical investigations of walnut fruit green husk extract as effective-biodegradable corrosion mitigating materials of a steel electrode in $1 \mathrm{M} \mathrm{HCl}$ electrolyte. J. Mol. Liq. 2021, 338, 116550. https://doi.org/10.1016/j.molliq.2021.116550

32. Elyor Berdimurodov, Abduvali Kholikov, Khamdam Akbarov, Lei Guo, Inhibition properties of 4,5dihydroxy-4,5-di-p-tolylimidazolidine-2-thione for use on carbon steel in an aggressive alkaline medium with chloride ions: Thermodynamic, electrochemical, surface and theoretical analyses. J. Mol. Liq. 2021, 327, 114813. https://doi.org/10.1016/j.molliq.2020.114813

33. Dakeshwar Kumar Verma, Mohsin Kazi, Mohammed S. Alqahtani, Rabbani Syed, Elyor Berdimurodov, Savas Kaya, Rajae Salim, Ashish Asatkar, Rajesh Haldhar, N-hydroxybenzothioamide derivatives as green and efficient corrosion inhibitors for mild steel: Experimental, DFT and MC simulation approach, J. Mol. Struct. 2021, 1241, 130648. https://doi.org/10.1016/j.molstruc.2021.130648 
34. Elyor Berdimurodov, Abduvali Kholikov, Khamdam Akbarov, Lei Guo, Aboubakr M. Abdullah, Mustafa Elik, A gossypol derivative as an efficient corrosion inhibitor for St2 steel in $1 \mathrm{M} \mathrm{HCl}+1 \mathrm{M} \mathrm{KCl}: \mathrm{An}$ experimental and theoretical investigation. J. Mol. Liq. 2021, 328, 115475. https://doi.org/10.1016/j.molliq.2021.115475

35. Mohamed Rbaaa, Asmaa Oubihi, Halima Hajji, Burak Tüzünd, Abdelhadi Hichar, El Hassane Anouar, Elyor Berdimurodov, Mohammed Aziz Ajana, Abdelkader Zarrouk, Brahim Lakhrissi, Synthesis, bioinformatics and biological evaluation of novel pyridine based on 8-hydroxyquinoline derivatives as antibacterial agents: DFT, molecular docking and ADME/T studies. J. Mol. Struct. 2021, 1244, 130934. https://doi.org/10.1016/j.molstruc.2021.130934

36. Elyor Berdimurodov, Abduvali Kholikov, Khamdam Akbarov, I.B. Obot, Lei Guo, Thioglycoluril derivative as a new and effective corrosion inhibitor for low carbon steel in a $1 \mathrm{M} \mathrm{HCl}$ medium: Experimental and theoretical investigation. J. Mol. Struct. 2021, 1234, 130165. https://doi.org/10.1016/j.molstruc.2021.130165

37. Dilfuza Nuriddinova, Farkhod Yusupov, Elyor Berdimurodov, Normahmat Yodgorov and Murod Mamanazarov, Adsorption Equilibrium, Kinetics, Thermodynamics and Dynamic Separation of Magnesium and Calcium Ions from Industrial Wastewater by New Strong Acid Cation Resin of SPVC. Pak. J. Anal. Environ. Chem. 2021, 22, No. 1, 127 - 138. http://doi.org/10.21743/pjaec/2021.06.13

38. E. Berdimurodov, A. Kholikov, K. Akbarov, L. Guo, Experimental and theoretical assessment of new and eco-friendly thioglycoluril derivative as an effective corrosion inhibitor of St2 steel in the aggressive hydrochloric acid with sulfate ions. J. Mol. Liq. 2021, 335, 116168. https://doi.org/10.1016/j.molliq.2021.116168 
Supplementary information

Table S1. Optimized structural parameters, such as bond lengths $(\AA)$ and bond angles $\left(^{\circ}\right)$, calculated by DFT method using 6-31G $(\mathrm{d}$, p) basis set.

\begin{tabular}{|c|c|c|c|c|c|c|c|c|c|c|}
\hline Row & Symbol & NA & NB & $\mathrm{NC}$ & Bond & Angle & Dihedral & $\mathbf{X}$ & $\mathbf{Y}$ & $\mathbf{Z}$ \\
\hline 1 & $\mathrm{C}$ & & & & & & & -3.2783120 & -0.7559330 & -1.1008590 \\
\hline 2 & $\mathrm{O}$ & 1 & & & 1.4385228 & & & -4.4641790 & -0.0837660 & -0.6412300 \\
\hline 3 & $\mathrm{C}$ & 2 & 1 & & 1.3554706 & 116.2666027 & & -5.0766240 & -0.6212000 & 0.4419950 \\
\hline 4 & $\mathrm{O}$ & 3 & 2 & 1 & 1.2126410 & 123.7209887 & -0.0281021 & -4.6787690 & -1.6113120 & 1.0180920 \\
\hline 5 & $\mathrm{C}$ & 3 & 2 & 1 & 1.5085513 & 110.6443464 & 179.9364775 & -6.2990240 & 0.1806210 & 0.8142160 \\
\hline 6 & $\mathrm{H}$ & 5 & 3 & 2 & 1.0893172 & 109.4772468 & 178.5361967 & -6.7902660 & -0.2819320 & 1.6693990 \\
\hline 7 & $\mathrm{H}$ & 5 & 3 & 2 & 1.0936427 & 110.1159051 & 57.2975832 & -6.9878850 & 0.2316850 & -0.0336740 \\
\hline 8 & $\mathrm{H}$ & 5 & 3 & 2 & 1.0939129 & 109.7600666 & -60.5976132 & -6.0080970 & 1.2063500 & 1.0589340 \\
\hline 9 & $\mathrm{C}$ & 1 & 2 & 3 & 1.5223787 & 112.4022912 & -82.6639080 & -2.0389590 & -0.3471340 & -0.3169430 \\
\hline 10 & $\mathrm{H}$ & 1 & 2 & 3 & 1.0920528 & 105.0911479 & 158.0779314 & -3.1685500 & -0.4565630 & -2.1453250 \\
\hline 11 & $\mathrm{H}$ & 1 & 2 & 3 & 1.0928584 & 109.4933029 & 39.5919071 & -3.4208690 & -1.8370660 & -1.0289680 \\
\hline 12 & $\mathrm{C}$ & 9 & 1 & 2 & 1.5351724 & 111.2417879 & 174.2129606 & -0.8187030 & -1.1816760 & -0.7307900 \\
\hline 13 & $\mathrm{C}$ & 12 & 9 & 1 & 1.5310359 & 110.9698985 & 173.3962403 & 0.4550410 & -0.6593880 & -0.0608180 \\
\hline 14 & $\mathrm{H}$ & 12 & 9 & 1 & 1.0967664 & 109.5029793 & 53.8601046 & -0.6859090 & -1.1220050 & -1.8178510 \\
\hline 15 & $\mathrm{H}$ & 12 & 9 & 1 & 1.0950121 & 110.7567937 & -64.9941677 & -0.9611700 & -2.2347910 & -0.4667760 \\
\hline 16 & $\mathrm{C}$ & 13 & 12 & 9 & 1.5273285 & 109.7741345 & -49.6306999 & 0.5604220 & 0.8514660 & -0.2581690 \\
\hline 17 & $\mathrm{C}$ & 16 & 13 & 12 & 1.5398306 & 111.0425653 & 52.8134985 & -0.7281170 & 1.5599500 & 0.1987730 \\
\hline 18 & $\mathrm{O}$ & 17 & 16 & 13 & 1.4257812 & 107.5572524 & -59.3800644 & -1.8045620 & 1.0366880 & -0.5760270 \\
\hline 19 & $\mathrm{H}$ & 9 & 1 & 2 & 1.1012842 & 108.6252719 & 53.7362262 & -2.2367860 & -0.4954640 & 0.7562250 \\
\hline 20 & $\mathrm{O}$ & 16 & 13 & 12 & 1.4416089 & 107.4037761 & 174.1707360 & \begin{tabular}{|l|}
1.6952780 \\
\end{tabular} & 1.3078570 & 0.5047500 \\
\hline 21 & $\mathrm{H}$ & 16 & 13 & 12 & 1.0943524 & 109.7500686 & -67.1847595 & 0.7239450 & 1.0756180 & -1.3167640 \\
\hline 22 & $\mathrm{C}$ & 20 & 16 & 13 & 1.3559395 & 117.1900475 & 149.2675625 & 2.3667400 & 2.3865820 & 0.0313970 \\
\hline 23 & $\mathrm{C}$ & 22 & 20 & 16 & 1.5035471 & 110.9655264 & -174.4608170 & 3.5953960 & 2.6590300 & 0.8540940 \\
\hline 24 & $\mathrm{H}$ & 23 & 22 & 20 & 1.0936257 & 109.9136307 & -54.5024590 & \begin{tabular}{|l|}
3.3231600 \\
\end{tabular} & 2.7579580 & 1.9086640 \\
\hline 25 & $\mathrm{H}$ & 23 & 22 & 20 & 1.0892963 & 109.5259625 & -175.6711066 & 4.0738800 & 3.5722520 & 0.5024620 \\
\hline 26 & $\mathrm{H}$ & 23 & 22 & 20 & 1.0937747 & 108.7782846 & 63.1821303 & 4.2750720 & 1.8067370 & 0.7647620 \\
\hline 27 & $\mathrm{O}$ & 22 & 20 & 16 & 1.2158553 & 123.4883650 & 4.9113934 & \begin{tabular}{|l|}
2.0099600 \\
\end{tabular} & 3.0260100 & -0.9392440 \\
\hline 28 & $\mathrm{O}$ & 13 & 12 & 9 & 1.4276923 & 108.4469621 & -170.4625490 & 1.5770180 & -1.3199700 & -0.6465690 \\
\hline 29 & $\mathrm{H}$ & 13 & 12 & 9 & 1.0974698 & 110.1360084 & 69.7889981 & 0.4287300 & -0.8691540 & 1.0160970 \\
\hline 30 & $\mathrm{O}$ & 17 & 16 & 13 & 1.3817132 & 113.2863926 & 179.2438233 & -0.6765040 & 2.9332680 & 0.0557170 \\
\hline 31 & $\mathrm{H}$ & 30 & 17 & 16 & 0.9711535 & 108.8899360 & 28.0705194 & -0.0743640 & 3.1509080 & -0.6744880 \\
\hline
\end{tabular}




\begin{tabular}{|c|c|c|c|c|c|c|c|c|c|c|}
\hline Row & Symbol & $\mathbf{N A}$ & NB & $\mathbf{N C}$ & Bond & Angle & Dihedral & $\mathbf{X}$ & $\mathbf{Y}$ & $\mathbf{Z}$ \\
\hline 32 & $\mathrm{H}$ & 17 & 16 & 13 & 1.1026388 & 109.9953729 & 59.2612483 & -0.9164370 & 1.3494820 & 1.2646300 \\
\hline 33 & $\mathrm{Si}$ & 28 & 13 & 12 & 1.6467518 & 127.2828657 & -148.2365313 & 3.0004600 & -1.7071890 & 0.0853230 \\
\hline 34 & $\mathrm{O}$ & 33 & 28 & 13 & 1.6444926 & 106.4920023 & 29.4067225 & 2.6572280 & -1.9490210 & 1.6753120 \\
\hline 35 & $\mathrm{O}$ & 33 & 28 & 13 & 1.6524865 & 116.9091072 & -91.3913805 & 4.2180700 & -0.5941660 & -0.0112030 \\
\hline 36 & $\mathrm{O}$ & 33 & 28 & 13 & 1.6493388 & 105.6241714 & 153.0164106 & 3.5773380 & -3.0189920 & -0.7311960 \\
\hline 37 & $\mathrm{H}$ & 35 & 33 & 28 & 0.9647566 & 113.3107650 & -92.1863178 & 4.8030770 & -0.7327700 & -0.7657300 \\
\hline 38 & $\mathrm{H}$ & 34 & 33 & 28 & 0.9633662 & 115.5766511 & -157.5763197 & 3.4001810 & -1.8512170 & 2.2807290 \\
\hline 39 & $\mathrm{H}$ & 36 & 33 & 28 & 0.9637489 & 114.9804114 & -33.1365522 & 2.9016580 & -3.6160720 & -1.0714370 \\
\hline
\end{tabular}

